engrafted into nonhuman primate hearts remains to be determined.

Despite these issues, the pioneering studies of Blin et al. are very encouraging. Their imaginative work using a nonhuman primate model of myocardial infarction to test the capacity of SSEA- $1^{+}$CPCs to engraft and differentiate into matured cardiomyocytes represents an important milestone. Future studies on the long-term survival, functional integration, physiological compatibility of engrafted cells, and beneficial effects on cardiac function will provide new insights into the potential use of SSEA- $1^{+}$CPCs for cardiovascular regenerative medicine. Most importantly, the ability to isolate nonhuman primate CPCs using a cell surface marker brings us one step closer to the ultimate dream of cell-based therapies for some of the most devastating forms of heart disease.

Address correspondence to: Deepak Srivastava, Gladstone Institute of Cardiovascular Disease, University of California, 1650 Owens Street, San Francisco, CA 94158.
Phone: 415.734.2716; Fax: 415.355.0141; E-mail: dsrivastava@gladstone.ucsf.edu.

1. Murry CE, Keller G. Differentiation of embryonic stem cells to clinically relevant populations: lessons from embryonic development. Cell. 2008;132(4):661-680.

2. Srivastava D, Ivey KN. Potential of stem-cellbased therapies for heart disease. Nature. 2006; 441(7097):1097-1099.

3. Passier R, van Laake LW, Mummery CL. Stem-cellbased therapy and lessons from the heart. Nature. 2008; 453(7193):322-329

4. Yi BA, Wernet O, Chien KR. Pregenerative medicine: developmental paradigms in the biology of cardiovascular regeneration. J Clin Invest. 2010; 120(1):20-28.

5. Kattman SJ,Huber TL, Keller GM. Multipotent flk-1+ cardiovascular progenitor cells give rise to the cardiomyocyte, endothelial, and vascular smooth muscle lineages. Dev Cell. 2006;11(5):723-732.

6 . Yang L, et al. Human cardiovascular progenitor cells develop from a $\mathrm{KDR}+$ embryonic-stem-cell-derived population. Nature. 2008;453(7194):524-528.

7. Bu L, et al. Human ISL1 heart progenitors generate diverse multipotent cardiovascular cell lineages. Nature. 2009;460(7251):113-117.

8. Laugwitz KL, et al. Postnatal isl1+ cardioblasts enter fully differentiated cardiomyocyte lineages. Nature. 2005;433(7026):647-653.

9. Moretti A, et al. Multipotent embryonic isl1+ progenitor cells lead to cardiac, smooth muscle, and endothelial cell diversification. Cell. 2006; 127(6):1151-1165.

10. Wu SM, et al. Developmental origin of a bipotential myocardial and smooth muscle cell precursor in the mammalian heart. Cell. 2006;127(6):1137-1150.

11. Blin G, et al. A purified population of multipotent cardiovascular progenitors derived from primate pluripotent stem cells engrafts in postmyocardial infarcted nonhuman primates. $J$ Clin Invest. 2010;120(4):1125-1139.

12. Park IH, et al. Reprogramming of human somatic cells to pluripotency with defined factors. Nature. 2008;451(7175):141-146.

13. Takahashi $\mathrm{K}$, et al. Induction of pluripotent stem cells from adult human fibroblasts by defined factors. Cell. 2007;131(5):861-872.

14. Takahashi K, Yamanaka S. Induction of pluripotent stem cells from mouse embryonic and adult fibroblast cultures by defined factors. Cell. 2006; 126(4):663-676.

15. Yu J, et al. Induced pluripotent stem cell lines derived from human somatic cells. Science. 2007; 318(5858):1917-1920.

16. van Laake LW, et al. Human embryonic stem cellderived cardiomyocytes survive and mature in the mouse heart and transiently improve function after myocardial infarction. Stem Cell Res. 2007; $1(1): 9-24$.

17. Laflamme MA, et al. Cardiomyocytes derived from human embryonic stem cells in pro-survival factors enhance function of infarcted rat hearts. Nat Biotechnol. 2007;25(9):1015-1024.

\title{
Prevention trumps treatment of antibody-mediated transplant rejection
}

\author{
Stuart J. Knechtle, Jean Kwun, and Neal Iwakoshi
}

Emory University School of Medicine, Atlanta, Georgia.

\begin{abstract}
Belying the spectacular success of solid organ transplantation and improvements in immunosuppressive therapy is the reality that long-term graft survival rates remain relatively unchanged, in large part due to chronic and insidious alloantibody-mediated graft injury. Half of heart transplant recipients develop chronic rejection within 10 years - a daunting statistic, particularly for young patients expecting to achieve longevity by enduring the rigors of a transplant. The current immunosuppressive pharmacopeia is relatively ineffective in preventing late alloantibody-associated chronic rejection. In this issue of the JCI, Kelishadi et al. report that preemptive deletion of $B$ cells prior to heart transplantation in cynomolgus monkeys, in addition to conventional posttransplant immunosuppressive therapy with cyclosporine, markedly attenuated not only acute graft rejection but also alloantibody elaboration and chronic graft rejection. The success of this preemptive strike implies a central role for $B$ cells in graft rejection, and this approach may help to delay or prevent chronic rejection after solid organ transplantation.
\end{abstract}

\section{Acute and chronic rejection}

Newly transplanted organs are susceptible within a week to acute rejection, medi-

Conflict of interest: Stuart J. Knechtle acknowledges stock ownership in Bristol-Myers Squibb.

Citation for this article: J Clin Invest. 2010; 120(4):1036-1309. doi:10.1172/JCI42532. ated dominantly by $\mathrm{T}$ cells, but are usually effectively protected from this form of inflammation and injury by currently used immunosuppressive agents such as calcineurin inhibitors, antiproliferative agents, mTOR inhibitors, and prophylactic therapy with $\mathrm{T}$ cell-specific antibodies. When acute rejection occurs, as it does in $5 \%-25 \%$ of solid organ recipients within the first year, it can typically be successfully treated with steroid therapy or, if needed, $\mathrm{T}$ cell-specific antibodies. However, the current immunosuppressive pharmacopeia is relatively ineffective in preventing or treating rejection mediated by $\mathrm{B}$ cells and the antibodies they produce. Antibody-mediated allograft injury, which occurs in $50 \%$ of heart transplant patients within 10 years, typically manifests more than a year after transplantation, more insidiously than $\mathrm{T}$ cell-mediated injury, and in a process characterized by complement deposition and microvascular obliteration that leads to tissue ischemia and eventually fibrosis with loss of graft function. Chronic graft rejection refers to this antibody-mediated process.

While factors contributing to chronic injury of organ transplants are multiple and include ischemia/reperfusion injury, preexisting donor disease, drug toxicities, and recurrence of original disease, the 


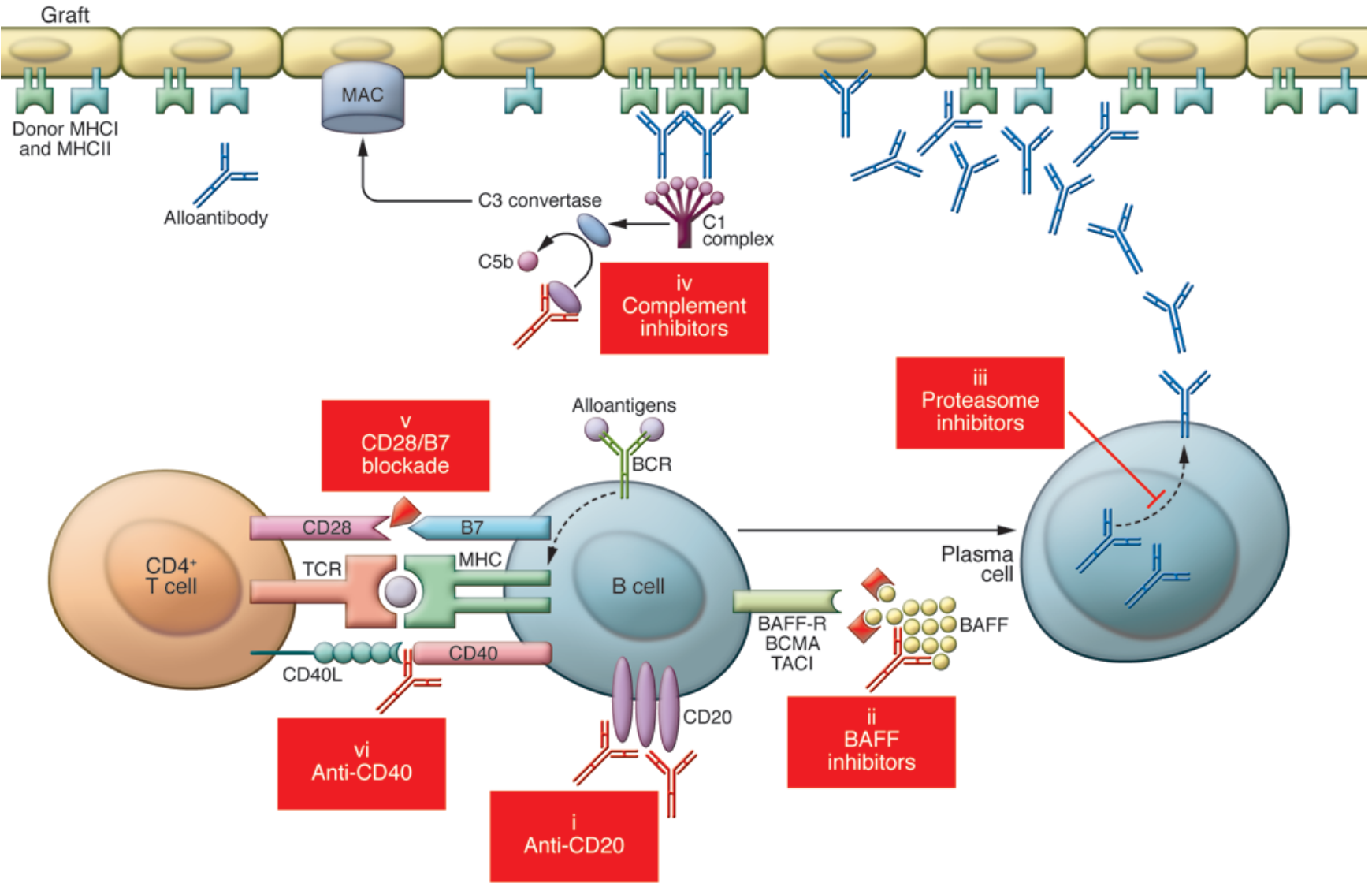

\section{Figure 1}

$\mathrm{B}$ cell- and antibody-related biologics in transplantation. (i) CD20-specific mAb (i.e., rituximab) (anti-CD20), as reported in the current issue of the $\mathrm{JCl}$ by Kelishadi et al (7), binds and selectively depletes $\mathrm{CD} 20^{+} \mathrm{B}$ cells, thereby reducing alloantibody levels. Third generation CD20-specific mAbs are under development (e.g., ocrelizumab, ofatumumab). (ii) Inhibitors such as belimumab neutralize BAFF, while inhibitors such as atacicept (TACI-Ig) inhibit both BAFF and APRIL. (iii) Proteasome inhibitors (e.g., bortezomib) reversibly bind to the proteasome and disrupt various cell signaling pathways including the NF-кB pathway. (iv) Complement inhibitors, such as eculizumab (an antibody specific for complement component 5 [C5]), bind the complement protein $\mathrm{C} 5$, leading to cessation of complement-mediated cell lysis via the membrane attack complex (MAC). Since activation of the complement system is initiated by binding of 2 alloantibody molecules to a multivalent antigen followed by formation of the $\mathrm{C} 1$ complex, $\mathrm{C} 1$ inhibitor $(\mathrm{C} 1-\mathrm{INH})$ prevents initiation of the serial complement cascade by inhibiting proteolytic cleavage of later complement components (specifically C2 and C4) and formation of C3 convertase. (v) Abatacept and belatacept (LEA29Y) are CTLA4-Ig molecules that bind the B7 costimulation molecule and block T cell costimulation of B cell activation and thereby production of alloantibodies. (vi) CD40-specific mAb (anti-CD40) binds the CD40 costimulation molecule. Blocking CD40L/CD40 interactions with CD40-specific antibody prevents $T$ cell help to $B$ cell activation, and consequently alloantibody production is inhibited.

subtle development in the graft recipient of antibodies specific for the foreign donor tissue (alloantibodies) in the months and years following organ transplantation has been shown to be an accurate predictor of graft failure $(1,2)$. The magnitude of this problem is compounded by the practical difficulties in designing feasible clinical trials to evaluate methods for preventing alloantibody development and by the paucity of proven strategies to prevent alloantibody development in large animal models or humans. Nevertheless, data suggest that if preexisting alloantibody levels can be reduced, the risk of graft loss is lower (3).

\section{B cell depletion as treatment for established antibody- mediated rejection}

In the medical literature, organ transplant patients experiencing antibody-mediated rejection have been treated with rituximab (a CD20-specific monoclonal antibody that depletes the $\mathrm{B}$ cell population) or by targeting of their plasma cells (antibody-secreting differentiated B cells), and in most cases these patients possessed preexisting alloantibody or suffered from early antibody-mediated rejection $(4,5)$. As expected, it is difficult to reverse the damage done by alloantibody in the setting of an established
B cell immune response, and the efficacy of targeting $B$ cells with rituximab under these posttransplant circumstances has been difficult to clearly establish. The combination of $\mathrm{B}$ cell depletion with profound $\mathrm{T}$ cell immunosuppression may also be complicated by loss of protective immunity (6). In other words, infection or malignancy may ensue, especially when both T cell- and B cell-depleting antibodies are administered simultaneously or sequentially. Therefore, an alternative strategy, that being prevention as opposed to treatment of the B cell alloimmune response, even if resorting to B cell depletion, may be attractive. 


\section{Preemptive B cell depletion}

In their study in this issue of the JCI, Kelishadi et al. (7) show that preemptive treatment of cynomolgus monkeys transplanted with an allogeneic heart with rituximab on the day of the transplant substantially eliminated the injury attributable to $B$ cells. In particular, infiltration of the graft by $\mathrm{B}$ cells was markedly reduced, as were intragraft levels of B cell-activating factor (BAFF; also known as B lymphocyte survival factor [BlyS]) and the B cell costimulatory molecules CD80 and CD86. In addition, the downstream effects of $\mathrm{B}$ cell activation were attenuated; for example, levels of alloantibody in the blood were reduced and less complement deposition in the graft was observed. Perhaps most importantly, these mechanistic changes were reflected by a substantial improvement in the microvascular integrity of the transplanted hearts (i.e., there was less chronic allograft vasculopathy) and by improved cardiac function, with four of four hearts beating well by 90 days compared with only three of seven in cynomolgus monkeys treated with cyclosporine alone. As seen from the control animals treated with cyclosporine alone, calcineurin inhibitors alone were unable to effectively prevent the injury inflicted by B cell-mediated rejection. Cotreatment with rituximab and cyclosporine also effectively prevented acute rejection compared with cyclosporine treatment alone, suggesting a role for $\mathrm{B}$ cells in acute rejection as well as chronic rejection.

\section{Implications for human transplant patients}

Therapeutic targeting of CD20 in transplantation may be appealing because of CD20's stable expression primarily on $\mathrm{B}$ cells in the peripheral blood and its absence from plasma cells, pro-B cells, and hematopoietic stem cells, thus permitting maintenance of serum IgG levels and posttreatment recovery by spared pro-B and stem cells (8). For the same reasons, therapeutic targeting of CD20 may not be as effective in treating recipients known to have donor-specific alloantibody prior to transplantation, since memory B cells and plasma cells capable of producing antibody specific for the donor organ would already be primed. Since many $T$ cell-mediated immune responses include a B cell component, the impact of $\mathrm{B}$ cell depletion may extend beyond suppression of measurable antibody (9), as is suggested by the observation in the current study that acute rejec- tion was reduced from a $57 \%$ incidence in cynomolgus monkeys treated with cyclosporine alone to zero by addition of rituximab to the treatment regimen (7).

Nonhuman primate (NHP) models, such as the one used by Kelishadi et al. (7), are far closer, genetically, to the human condition than any rodent model might be, and thus the current report is expected to predict better than any rodent model of transplantation how humans might respond to B cell depletion. Nevertheless, it is worth noting that even observations in NHPs in the field of organ transplantation have sometimes been difficult to translate directly into the clinic $(10,11)$. By analogy, human heart transplant patients usually receive three or four simultaneous immunosuppressive agents to prevent $\mathrm{T}$ cell-mediated rejection, whereas the cynomolgus monkeys in the study by Kelishadi et al. received high-dose cyclosporine as their sole immunosuppressive agent (7). The applicability of the findings of the current study to human organ transplantation will therefore require rigorous testing in order to determine whether preemptive CD20 monoclonal antibody treatment in the setting of more intense $\mathrm{T}$ cell immunosuppression is accompanied by opportunistic infection.

\section{Other B cell strategies for transplantation}

Targeting B cell immunity without depleting these cells in order to prevent alloantibody development may also lead to opportunities to prevent allograft injury (Figure 1). Such strategies include targeting complement pathway components (12) and B cell cytokines and/or chemokines such as $\mathrm{BAFF}$ and/or a proliferation-inducing ligand (APRIL), which may influence both $\mathrm{B}$ and $\mathrm{T}$ cell responses $(13,14)$. Other biologics being considered for development for the targeting of $\mathrm{B}$ cell responses in the setting of transplantation are those that affect the costimulatory pathways. Interactions between $\mathrm{CD} 28$ on $\mathrm{CD}^{+}$ $\mathrm{T}$ cells and CD80/CD86 on B cells, as well as between CD40 ligand (CD40L; also known as CD154) on activated CD4 ${ }^{+} \mathrm{T}$ cells and CD40 on B cells have been shown to participate in providing $\mathrm{T}$ cell help to B cells (15). The CD40/CD40L interaction stimulates B cell proliferation and isotype switching in the appropriate cytokine milieu $(16,17)$. CD28 and CTLA4 expression have also been shown to be involved in germinal center formation (18).
Each of these potential therapies is under active investigation. It will be important to compare the relative safety and efficacy of such strategies with that of profound B cell depletion with rituximab. Additionally, it will be necessary to determine the durability and need for repeated application of $B$ cell therapy in the setting of constant exposure to alloantigen, as is the case with an organ transplant. Nevertheless, the current report by Kelishadi et al. (7) offers clear experimental evidence in a large animal model that B cell targeting in parallel with $\mathrm{T}$ cell inhibition can prevent alloantibody development and lead to improved longterm graft histology and better small blood vessel patency. Prevention of chronic rejection would represent a major advance for the field of transplantation, and prevention of alloantibody development is more likely to succeed than are strategies to reverse ongoing antibody-mediated graft injury.

\section{Acknowledgments}

The authors are supported by NIH grant AI074635 (to S.J. Knechtle) and by Roche Organ Transplantation Research Foundation grant 962141545 (to N. Iwakoshi).

Address correspondence to: Stuart J. Knechtle, Emory University School of Medicine, 5105 WMB, 101 Woodruff Circle, Atlanta, GA 30322. Phone: 404.712.9910; Fax: 404.727.3660; E-mail: Stuart.Knechtle@ emoryhealthcare.org.

1. Terasaki PI. Humoral theory of transplantation. Am J Transplant. 2003;3(6):665-673.

2. Terasaki PI, Cai J. Human leukocyte antigen antibodies and chronic rejection: from association to causation. Transplantation. 2008;86(3):377-383.

3. Everly MJ, et al. Reducing de novo donor-specific antibody levels during acute rejection diminishes renal allograft loss. Am J Transplant. 2009;9(5):1063-1071.

4. Ramos EJ, et al. The effect of desensitization protocols on human splenic B-cell populations in vivo. Am J Transplant. 2007;7(2):402-407.

5. Zarkhin V, et al. A randomized, prospective trial of rituximab for acute rejection in pediatric renal transplantation. Am J Transplant. 2008;8(12):2607-2617.

6. Kamar $\mathrm{N}$, et al. Incidence and predictive factors for infectious disease after rituximab therapy in kidney-transplant patients. Am J Transplant. 2010; 10(1):89-98.

7. Kelishadi SS, et al. Preemptive CD20 ${ }^{+}$B cell depletion attenuates cardiac allograft vasculopathy in cyclosporine-treated monkeys. J Clin Invest. 2010; 120(4):1275-1284.

8. Teeling JL, et al. The biological activity of human CD20 monoclonal antibodies is linked to unique epitopes on CD20. J Immunol. 2006;177(1):362-371.

9. Pescovitz MD, et al. Rituximab, B-lymphocyte depletion, and preservation of beta-cell function. NEngl J Med. 2009;361(22):2143-2152.

10. Hale DA, Dhanireddy K, Bruno D, Kirk AD. Induction of transplantation tolerance in non-human primate preclinical models. Philos Trans R Soc Lond 
B Biol Sci. 2005;360(1461):1723-1737.

11. Knechtle SJ, Hamawy MM, Hu H, Fechner JH Jr, Cho CS. Tolerance and near-tolerance strategies in monkeys and their application to human renal transplantation. Immunol Rev. 2001;183:205-213.

12. Sacks S, Lee Q, Wong W, Zhou W. The role of complement in regulating the alloresponse. Curr Opin Organ Transplant. 2009;14(1):10-15.

13. Walters $\mathrm{S}$, et al. Increased CD4+Foxp $3+\mathrm{T}$ cells in BAFF-transgenic mice suppress $\mathrm{T}$ cell effector responses. J Immunol. 2009;182(2):793-801.

14. Bloom D, et al. BAFF is increased in renal transplant patients following treatment with alemtuzumab. Am J Transplant. 2009;9(8):1835-1845.

15. Larsen CP, et al. Rational development of LEA29Y (belatacept), a high-affinity variant of CTLA4-Ig with potent immunosuppressive properties. AmJ Transplant. 2005;5(3):443-453.
16. Li Y, Ma L, Yin D, Shen J, Chong AS. Long-term control of alloreactive $B$ cell responses by the suppression of T cell help. J Immunol. 2008;180(9):6077-6084.

17. Quezada SA, Jarvinen LZ, Lind EF, Noelle RJ. CD40/ $\mathrm{CD} 154$ interactions at the interface of tolerance and immunity. Annu Rev Immunol. 2004;22:307-328.

18. Ferguson SE, Han S, Kelsoe G, Thompson CB. CD28 is required for germinal center formation. J Immunol. 1996;156(12):4576-4581.

\title{
Pathogen-specific antibodies: codependent no longer
}

\author{
Edward N. Janoff',2 and Daniel N. Frank ${ }^{1,3}$
}

\begin{abstract}
${ }^{1}$ Mucosal and Vaccine Research Program Colorado. ${ }^{2}$ University of Colorado Denver School of Medicine, Department of Medicine, Division of Infectious Diseases, Aurora, and Department of Veterans Affairs Medical Center, Denver. ${ }^{3}$ Department of Molecular, Cellular, and Developmental Biology, University of Colorado Boulder.
\end{abstract}

\begin{abstract}
Antibody-mediated defense against pathogens typically requires complex interactions between antibodies and other constituents of the humoral and cellular immune systems. However, recent evidence indicates that some antibodies alone can inhibit pathogen function in the absence of complement, phagocytes, or NK cells. In this issue of the JCI, McClelland et al. have begun to elucidate the molecular bases by which antibodies alone can impact pathogen growth and metabolism. They show that mAbs specific for the polysaccharide capsule of the human pathogenic fungus Cryptococcus neoformans elicit diverse effects on fungal gene expression, lipid biosynthesis, susceptibility to amphotericin B, cellular metabolism, and protein phosphorylation. These data suggest that pathogens have the capacity to generate broad metabolic responses as a result of surface binding by pathogen-specific antibodies, effects that may hold therapeutic promise.
\end{abstract}

\section{Evolving concepts of antibody defense}

The word immunity derives from the Latin "immunitas" meaning exemption and has come to mean protection from disease. Immunity has been observed over the centuries during plagues in Athens and Byzantium, epidemics of bubonic plague and smallpox, as well as with snake bites and vaccinations (1). The identification of specific pathogens in the late 19th century was associated with the concept that such organisms were ultimately inhibited by depleting their environment of required nutrients, by their own metabolic by-products, or by the inhospitableness of infected tissues. Enter host defense. Initial conflicts arose between advocates of a predominantly soluble or humoral basis for immunity and those favoring a cellular basis. These disparate viewpoints were

Conflict of interest: Edward N. Janoff's laboratory has received research funding from Pfizer Pharmaceuticals (previously Wyeth Vaccines) and VaxInnate Corporation for vaccine studies unrelated to Cryptococcus.

Citation for this article: J Clin Invest. 2010; 120(4):1039-1042. doi:10.1172/JCI42695. ultimately reconciled in large part when antibodies, the key mediators of humoral immunity, were shown to rely on other soluble factors, particularly complement, and cells known as phagocytes to provide protection against and mediate resolution of infection. For its part, the microbe itself often expresses a range of protective defenses. These microbial virulence factors may bind, mask, or degrade complement components; cleave adherent antibodies (e.g., IgA1 protease); or subvert the activity of antibodies by binding to their effector Fc constant regions (e.g., via staphylococcal protein A or streptococcal protein $\mathrm{G}$ ) that otherwise direct pathogens to an $\mathrm{Fc}$ receptor-bearing phagocyte. The protective effects of antibodies are classically mediated through their specificity for the pathogen (facilitated via their variable regions) and the ability of their Fc constant region to act as a bridge or scaffold. Other host defense mechanisms (e.g., complement, phagocytes, and NK cells) use this foundation to induce the fatal injuries on the pathogen, on which antibody defense is dependent (Figure 1A).
However, in their study in this issue of the JCI, McClelland et al. advance an intriguing conceptual paradigm that binding of specific antibodies alone can elicit a range of metabolic perturbations in the fungal pathogen Cryptococcus neoformans (2). C. neoformans, widespread in the environment, is well-controlled and rarely symptomatic in healthy individuals, in large part because of antibody-dependent mechanisms. However, the organism causes recalcitrant disease and high mortality in patients with advanced cell-mediated immunodeficiency, such as those individuals with HIV/AIDS who have very low $\mathrm{CD}^{+}$ $T$ cell counts and patients who have undergone solid organ transplantation $(3,4)$. McClelland and colleagues show that three antibodies that bind to distinct topological sites on the polysaccharide capsule of C. neoformans elicit varying effects on its gene expression (2). The effects are direct and due to the antibodies in the absence of other soluble or cellular host elements, providing evidence that pathogens can recognize and respond to antibody binding by modulating distinct microbial genetic pathways (Figure 1B). These findings raise the intriguing possibility that the physiology of a pathogen and its susceptibility to clearance may be manipulated by rational antibody design.

\section{Building on the past}

Previous studies have revealed that, independent of the presence of complement or phagocytes, antibody-pathogen interactions can disrupt microbial integrity, although the genetic mechanism(s) remained undetermined (5-14). Antibodies 